\title{
Evidence for gravitational-wave dominated emission in the central engine of short GRB 200219A
}

\author{
Hou-Jun L ̈̈̈ ${ }^{1}$, Yong Yuan ${ }^{1}$, Lin Lan ${ }^{2}$, Bin-Bin Zhang ${ }^{3}$, Jin-Hang Zou ${ }^{4}$, Zong-Kai Peng ${ }^{3}$, \\ Jun Shen ${ }^{1}$, Yun-Feng Liang ${ }^{1}$, Xiang-Gao Wang ${ }^{1}$, and En-Wei Liang ${ }^{1}$
}

\begin{abstract}
GRB 200219A is a short gamma-ray burst (GRB) with an extended emission (EE) lasting $\sim 90$ s. By analyzing data observed with the Swift/BAT and Fermi/GBM, we find that a cutoff power-law model can adequately fit the spectra of the initial short pulse with $\mathrm{E}_{\mathrm{p}}=1387_{-134}^{+232} \mathrm{keV}$. More interestingly, together with the EE component and early X-ray data, it exhibits plateau emission smoothly connected with a $\sim t^{-1}$ segment and followed by an extremely steep decay. The short GRB composed of those three segments is unique in the Swift era and is very difficult to explain with the standard internal/external shock model of a black hole central engine, but could be consistent with the prediction of a magnetar central engine from the merger of an NS binary. We suggest that the plateau emission followed by a $\sim t^{-1}$ decay phase is powered by the spin-down of a millisecond magnetar, which loses its rotation energy via GW quadrupole radiation. Then, the abrupt drop decay is caused by the magnetar collapsing into a black hole before switching to EM-dominated emission. This is the first short GRB for which the X-ray emission has such an intriguing feature powered by a magnetar via GW-dominated radiation. If this is the case, one can estimate the physical parameters of a magnetar, the GW signal powered by a magnetar and the merger-nova emission are also discussed.
\end{abstract}

Subject headings: Gamma-ray bursts; Gravitational waves;

\footnotetext{
${ }^{1}$ Guangxi Key Laboratory for Relativistic Astrophysics, School of Physical Science and Technology, Guangxi University, Nanning 530004, China; lhj@gxu.edu.edu

${ }^{2}$ Department of Astronomy, Beijing Normal University, Beijing, China

${ }^{3}$ Key Laboratory of Modern Astronomy and Astrophysics (Nanjing University), Ministry of Education, Nanjing 210093, China; bbzhang@nju.edu.cn

${ }^{4}$ Department of Space Sciences and Technology, Hebei Normal University, Shijiazhuang 050024, China
} 


\section{Introduction}

The progenitors of short gamma-ray bursts (GRBs) are thought to be from compact star mergers (Kumar \& Zhang 2015, for a review), such as neutron star-neutron star mergers (NS-NS, Paczynski 1986; Eichler et al. 1989), or neutron star-black hole mergers (NS-BH, Paczynski 1991). Moreover, such coalescence systems are also the main targets as a strong source of gravitational waves (GWs, Berger 2014, for a review). The first directly detection of GW event (GW 170817) associated with short GRB 170817A was achieved by Advanced LIGO and Virgo (Abbott et al. 2017a, b; Goldstein et al. 2017; Savchenko et al. 2017; Zhang et al. 2018a), and it opened a new window to study the properties for such a catastrophic crash (Abbott et al. 2017a).

The remnant of $\mathrm{NS}-\mathrm{BH}$ mergers must be a $\mathrm{BH}$ surrounded by an accretion torus. NS-NS mergers, on the other hand, may result in a BH (Popham et al. 1999; Wheeler et al. 2000; Lei et al. 2009; Liu et al. 2017), or a rapidly spinning, strongly magnetized NS (known as a millisecond magnetar, Usov 1992; Thompson 1994; Dai \& Lu 1998a, b;; Zhang \& Mészáros 2001; Metzger et al. 2011; Bucciantini et al. 2012; Lü et al. 2015; Chen et al. 2017). From the theoretical point of view, depending on the nascent NS mass and poorly know equation of state of NS, the magnetar can survive from milliseconds to several days (Lasky et al. 2014; Li et al. 2016; Gao et al. 2016; Lü et al. 2018). Based on the lifetime of the magnetar, three types of magnetars can be classified, namely hypermassive NS, which is supported by differential rotation with 10-100 ms lifetime before collapsing into a BH (Rosswog et al. 2003) , supramassive NS, which is supported by rigid rotation with survival from tens of seconds to thousands before collapsing to a BH (Rowlinson et al. 2010), and stable NS with much longer lifetimes (Dai et al. 2006; Gao \& Fan 2006; Zhang 2013; Kumar \& Zhang 2015).

From the observational point of view, the X-ray "internal plateau" (a nearly fairly constant emission followed by a steep decay with a decay slope $\alpha>3) \sqrt{1}$ in some short GRBs may be a "smoking gun" for a supramassive magnetar as the central engine (Rowlinson et al. 2010, 2013; Lü et al. 2015, 2017). Moreover, the extended emission (EE) of GRB prompt emission and shallow decay phase is naturally explained as the energy injection of the magnetar in GRB central engine (Dai \& Lu 1998a; Zhang \& Mészáros 2001; Troja et al. 2007; Lyons et al. 2010; Lü et al. 2015). The magnetar can be spun down by losing its rotation energy via GW radiation or magnetic dipole (MD) radiations (Dai \& Lu 1998a; Zhang \& Mészáros 2001; Corsi \& Mészáros 2009; Fan et al. 2013; Giacomazzo \& Perna 2013; Lasky et al. 2014; Metzger \& Piro 2014; Ravi \& Lasky 2014; Lü \& Zhang 2014; Sowell et al. 2019). Lasky \& Glampedakis (2016) presented more details to derive the luminosity of X-ray

\footnotetext{
${ }^{1}$ Throughout the paper we adopt the convention $F_{\nu} \propto t^{-\alpha} \nu^{-\beta}$.
} 
evolution with time as $t^{-1}$ and $t^{-2}$ when the energy loss is dominated by GW and MD, respectively (also see Zhang \& Mészáros 2001). Lü et al. (2018) found out several long GRBs whose X-ray emissions are consistent with the above scenario. However, up to now, no directly observed evidence shows that the energy loss of supramassive magnetar from double NS mergers is dominated by GW quadrupole emission.

GRB 200219A is a short-hard burst without redshift measurement. Based on the properties of its multi-wavelength data presented here, the central engine of GRB 200219A seems to be a supramassive magnetar which originates from the merger of an NS binary. We consider that the magnetar lost its rotation energy via GW radiation before collapsing into $\mathrm{BH}$. We present our data reduction from Swift and Fermi observations in $\S 2$. In $\S 3$, we present the details of physical interpretation with a magnetar central engine for GRB 200219A. The calculations of GW radiation and possible merger-nova of magnetar are presented in $\S 4$ and $\S 5$. Conclusions are drawn in $\S 6$ with some additional discussion. Throughout the paper, a concordance cosmology with parameters $H_{0}=71 \mathrm{~km} \mathrm{~s}^{-1} \mathrm{Mpc}^{-1}, \Omega_{M}=0.30$, and $\Omega_{\Lambda}=0.70$ is adopted.

\section{Data reduction and analysis}

\subsection{Swift data reduction}

GRB 200219A triggered the Burst Alert Telescope (BAT) at 07:36:49 UT on 19 February 2020 (Lien et al. 2020). We downloaded the BAT data from the Swift website2, and use the standard HEASOFT tools (version 6.12) to process the BAT data, and running the late "convert" command from the HEASOFT software release to obtain the energy scale for the BAT events. The light curves in different energy bands and spectra are extracted by running batbinevt (Sakamoto et al. 2008). Then, we calculate the cumulative distribution of the source counts using the arrival time of a fraction between $5 \%$ and $95 \%$ of the total counts to define $T_{90}$. The time bin size is fixed to $128 \mathrm{~ms}$ in this case due to the short duration. The light curve shows a short-pulse with duration $T_{90} \sim 0.48 \mathrm{~s}$ (see Figure 1). The background is extracted using two time-intervals, one before and one after the burst. We model the background as Poisson noise, which is the standard background model for prompt emission in BAT events. We invoked Xspec to fit the spectra. For technical details, please refer to Sakamoto et al. (2008). The time-averaged spectrum of short-pulse is best fit by a simple power-law model with an index $0.76 \pm 0.08$. Moreover, Laha et al. (2020)

\footnotetext{
${ }^{2}$ https : //www.swift.ac.uk/archive/selectseq.php?source $=$ obs\&tid $=957271$
} 
report that the initial short-pulse seems to be followed by a soft EE component lasting $\sim 90$ $\mathrm{s}$, and maybe some even weaker emission until $\sim 300 \mathrm{~s}$. The X-ray Telescope (XRT) began observing the field 67 seconds after the BAT trigger (Lien et al. 2020). We made use of the public data from the Swift archive 3 (Evans et al. 2009). The Ultra-Violet Optical Telescope (UVOT, Roming et al. 2005) observed the field at $T_{0}+74 \mathrm{~s}$, but no optical afterglow was consistent with the XRT position (Siegel et al. 2020).

\subsection{Fermi data reduction}

The Fermi Gamma-ray Burst Monitor (GBM) was triggered and located GRB 200219A at 07:36:49.10 UT on 19 February 2020 (Bissaldi \& Meegan 2020). GBM has 12 sodium iodide (NaI) and two bismuth germanate (BGO) scintillation detectors are covering the energy range from $8 \mathrm{keV}$ to $40 \mathrm{MeV}$ (Meegan et al. 2009). We downloaded the corresponding Time-Tagged-Event data from the public data site of Fermi/GBM 4 . For more details of data reduction of light curve and spectra procedure refer to (Zhang et al. 2016). The light curves of $n 3$ and b0 detectors are shown in Figure 1, it consists of a single emission episode. We estimate $T_{90}$ of the burst according to the cumulative net count rate, and the duration of $90 \%$ total net counts is $T_{90} \sim 0.52 \mathrm{~s}$ in $50-300 \mathrm{keV}$ with starting and ending time $T_{90,1} \sim-0.02$ $\mathrm{s}$ and $T_{90,2} \sim 0.5$ (Figure 1). The EE component of the burst is not significant in the GBM temporal analysis.

We also extract the time-averaged spectrum of GRB 200219A between $T_{90,1}$ and $T_{90,2}$. The background spectra are extracted from the time intervals before and after the prompt emission phase and modeled with an empirical function (Zhang et al. 2011), and the spectral fitting is performed by using our automatic code "McSpecfit" in Zhang et al. (2018b). Several spectral models can be selected to test the spectral fitting of burst, such as power-law (PL), cutoff power-law (CPL), Band function (Band), Blackbody (BB), as well as combinations of any two models. Then, we compare the goodness of the fits and find that the CPL model is the best one to adequately describe the observed data by invoking the Bayesian Information Criteria (BIC) 5 . The CPL model fit is shown in Figure 2 for the count spectrum and photon spectrum, as well as parameter constraints of the fit. It gives peak energy $\mathrm{E}_{\mathrm{p}}=1387_{-134}^{+232}$

\footnotetext{
${ }^{3}$ https : //www.swift.ac.uk/xrt_curves/00957271

${ }^{4}$ https : //heasarc.gsfc.nasa.gov/FTP/fermi/data/gbm/daily/

${ }^{5}$ The BIC is a criterion for model selection among a finite set of models. The model with the lowest BIC is preferred. The BIC values of different model are presented as: 477(Band), 358(BB), 293(CPL), 505(PL), 369(Band+BB), 517(BB+PL), 304(BB+CPL).
} 
$\mathrm{keV}$, and a lower energy spectral index of $\Gamma_{\mathrm{ph}}=-0.65 \pm 0.07$. The best-fit parameters of CPL fits are listed in Table 1 . The estimated event fluence $\left(1-10^{4} \mathrm{keV}\right)$ in this time interval is $5.08_{-1.02}^{+1.27} \times 10^{-6} \mathrm{erg}^{\mathrm{cm}^{-2}}$.

\subsection{Statistical comparison of Burst and X-ray light-curve fits}

The observed properties of prompt emission for short GRB 200219A has a high peak energy and EE component. By comparing the $\mathrm{E}_{\mathrm{p}}$ value of GRB 200219A with that of other short GRBs observed by Fermi/GBM, we find that the $\mathrm{E}_{\mathrm{p}}$ of GRB 200219A is larger than that of most other short GRBs, but still falls in the typical range (see Figure 3). Moreover, no optical counterpart associated with GRB 200219A means that the redshift is unknown. In order to check whether GRB 200219A is an unusual event or not, we overplot GRB 200219A in the $E_{\mathrm{p}}-E_{\text {iso }}$ diagram (Amati et al. 2002; Zhang et al. 2009) with pseudo redshift from $z=0.01$ to $z=1$ in Figure 3. The data of Type I and Type II GRBs6, as well as fits are taken from Zhang et al. (2009). We find that it an outlier of the short GRB population for $z<0.5$, but is located well within the $1 \sigma$ region for $z>0.5$.

The X-ray light curve of GRB 200219A seems to be interesting, it is composed of several power law segments. Firstly, we extrapolate the BAT $(15-150 \mathrm{keV})$ data to the XRT band $(0.3-10 \mathrm{keV})$ by assuming a single power-law spectrum (O'Brien et al. 2006; Liang et al. 2008; Evans et al. 2009; Li et al. 2012). Then, we perform a empirical fit to the light curve with a smoothed triple power law model,

$$
F=\left(F_{1}^{-\omega_{2}}+F_{2}^{-\omega_{2}}\right)^{-1 / \omega_{2}}
$$

where $F_{1}$ and $F_{2}$ can be expressed as

$$
\begin{gathered}
F_{1}=F_{0}\left[\left(\frac{t}{t_{\mathrm{b}, 1}}\right)^{\omega_{1} \alpha_{1}}+\left(\frac{t}{t_{\mathrm{b}, 1}}\right)^{\omega_{1} \alpha_{2}}\right]^{-1 / \omega_{1}}, \\
F_{2}=F_{1}\left(t_{b, 2}\right)\left(\frac{t}{t_{b, 2}}\right)^{-\alpha_{3}},
\end{gathered}
$$

where $t_{b, 1}$ and $t_{b, 2}$ are the two break times, $\alpha_{1}, \alpha_{2}$, and $\alpha_{3}$ are the decay slopes before and after $t_{b, 1}$, and after $t_{b, 2}$, respectively. $\omega_{1}$ and $\omega_{2}$ describe the sharpness of the break at $t_{b, 1}$ and $t_{b, 2}$, and $\omega_{1}=\omega_{2}=3$ is fixed in our fits. The light curve fitting is shown in

6hang et al. (2009) proposed that Type I and Type II GRBs are originated from compact stars merger and massive star core-collapse, respectively. 
Figure 4, and fitting results are presented below, $t_{b, 1}=(57 \pm 18) \mathrm{s}, t_{b, 2}=(190 \pm 27) \mathrm{s}$, $\alpha_{1}=0.05 \pm 0.08, \alpha_{2}=1.18 \pm 0.15, \alpha_{3}=4.67 \pm 0.24, F_{0}=(5.08 \pm 1.16) \times 10^{-9} \mathrm{erg} \mathrm{cm}^{-2} \mathrm{~s}^{-1}$, and $\chi^{2} / d o f=72 / 54$. One basic question is that whether one power-law, or smooth broken power-law function can fit the the light curve well enough. In order to test that, we also adopted one power-law function to fit the data, and find that the $\chi^{2} / \operatorname{dof}=75 / 32$, and even for a smooth broken power-law function with $\chi^{2} / d o f=74 / 45$. So that, we adopted a smoothed triple power law model to fit the data and infer the physical parameters with the fitting results.

\section{Physical interpretation with magnetar central engine}

Magnetar as central engine of short GRBs are extensively discussed (Dai et al. 2006; Gao \& Fan 2006; Rowlinson et al. 2010; Metzger et al. 2011; Rowlinson et al. 2013; Kumar \& Zhang 2015; Lü et al. 2015). Considering a newly born magnetar, it is spun down via a combination of electromagnetic (EM) dipole and gravitational wave (GW) quadrupole emission (Shapiro \& Teukolsky 1983; Zhang \& Mészáros 2001),

$$
\begin{aligned}
-\frac{d E_{\mathrm{rot}}}{d t}=-I \Omega \dot{\Omega} & =L_{\mathrm{EM}}+L_{\mathrm{GW}} \\
& =\frac{B_{\mathrm{p}}^{2} R^{6} \Omega^{4}}{6 c^{3}}+\frac{32 G I^{2} \epsilon^{2} \Omega^{6}}{5 c^{5}}
\end{aligned}
$$

where $E_{\text {rot }}=\frac{1}{2} I \Omega^{2}$ is the rotation energy of magnetar, $I$ is the moment of inertia, $\Omega, P_{0}, B_{p}$, $\epsilon, R$, and $M$ are the angular frequency, rotating period, surface magnetic field, ellipticity, radius, and mass of the neutron star, respectively. The convention $Q=10^{x} Q_{x}$ is adopted in cgs units.

Lasky \& Glampedakis (2016) and Lü et al. (2018) derived the electromagnetic luminosity as function of time for both EM dipole and GW emission dominated energy loss, and the evolution behaviors are following,

$$
\begin{aligned}
& L_{\mathrm{EM}}(t)=L_{\mathrm{em}, 0}\left(1+\frac{t}{\tau_{\mathrm{c}, \mathrm{em}}}\right)^{-2},(\mathrm{EM} \text { dominated }) \\
& L_{\mathrm{EM}}(t)=L_{\mathrm{em}, 0}\left(1+\frac{t}{\tau_{\mathrm{c}, \mathrm{gw}}}\right)^{-1},(\mathrm{GW} \text { dominated })
\end{aligned}
$$

where $L_{\mathrm{em}, 0}$ is the initial kinetic luminosity,

$$
L_{\mathrm{em}, 0}=1.0 \times 10^{49} \mathrm{erg} \mathrm{s}^{-1}\left(B_{p, 15}^{2} P_{0,-3}^{-4} R_{6}^{6}\right)
$$


$\tau_{\mathrm{c}, \mathrm{em}}$ and $\tau_{\mathrm{c}, \mathrm{gw}}$ are the characteristic spin-down time scale for EM and GW dominated, respectively,

$$
\begin{gathered}
\tau_{\mathrm{c}, \mathrm{em}} \sim 2.05 \times 10^{3} \mathrm{~s}\left(I_{45} B_{p, 15}^{-2} P_{0,-3}^{2} R_{6}^{-6}\right), \\
\tau_{\mathrm{c}, \mathrm{gw}} \sim 9.1 \times 10^{3} \mathrm{~s}\left(I_{45}^{-1} \epsilon_{-3}^{-2} P_{0,-3}^{4}\right) .
\end{gathered}
$$

Moreover, one can obtain the transition time $\left(\tau_{*}\right)$ which point is from GW dominated to EM dominated emission (Zhang \& Mészáros 2001; Lasky \& Glampedakis 2016; Lü et al. 2018),

$$
\tau_{*}=\frac{\tau_{\mathrm{c}, \mathrm{em}}}{\tau_{\mathrm{c}, \mathrm{gw}}}\left(\tau_{\mathrm{c}, \mathrm{em}}-2 \tau_{\mathrm{c}, \mathrm{gw}}\right)
$$

The formation and evolution of magnetar central engine roughly can be described as following. A possible remnant of double NSs merger is supramassive NS that is supported by rigid ration if the equation of state of NS is stiff enough. The magnetar is going on losing its rotation energy via MD or GW radiations to result in the magnetar spin-down due to its strong surface magnetic filed and/or asymmetry of mass. If the energy loss of NS is initially dominated by MD radiation, the luminosity evolves as $L \propto \sim t^{-2}$, and it survives until is collapsed into black hole with a more steeper decay. Alternative, if the energy loss of NS is initially dominated by GW radiation, the luminosity evolves as $L \propto \sim t^{-1}$ until MD dominated with $\sim t^{-2}$, or with steeper decay when it collapse into black hole before switching the MD radiation.

Motivated by the above derivations, we find that the X-ray evolution behavior of GRB 200219A is consistent with the magnetar central engine. The physical process can be described as follows: the progenitor of short GRB 200219A originated from double NSs merger, a supramassive NS is produced after the merger (if the masses distribution of the two NSs are perfection, or its equation of state is stiff enough), accretion of the torus material into the NS may launch a jet, which powers a short-duration GRB (the prompt emission of short GRB). The observed EE component or plateau emission is from the energy injection of magnetar dipole radiation before the NS spin-down. After tens of seconds, the newborn NS is spun down by losing its rotation energy via GW emission (the observed $\sim t^{-1}$ segment), and then survive for hundreds of seconds before collapsing to a $\mathrm{BH}$ (even steeper segment), but is not enough time to switch into EM dominated phase. Moreover, a normal decay segment with slope $\sim 1$ at the later time is consistent with external shock model, this component is the afterglow emission from the jet.

If this is the case, we find that the GRB 200219A possibly presents the first indirect evidence to show the GW-dominated emission of supramassive NS in the central engine, this can be confirmed by systematically searching for all short GRBs observed by 
Swift/BAT. However, with no measured redshift it is difficult to evaluate the properties of supramassive magnetar; there pseudo redshift values are adopted in our calculations. One is $z=0.01$ (corresponding to $\sim 40 \mathrm{Mpc}$ luminosity distance), that of GW170817/GRB 170817A (Abbott et al. 2017a). Another one is $z=0.1$ (corresponding to $\sim 450 \mathrm{Mpc}$ luminosity distance), which is close to the upper limit of the GW signal detected by LIGO/Vergo (Abbott et al. 2017b). The third one is $z=0.5$ the central value of redshift distribution for all short GRBs with z measurements (Lü et al. 2015). Based on the derivations of above, one has $\tau_{\mathrm{c}, \mathrm{gw}} \simeq t_{b, 1} /(1+z)$, and $\tau_{*}>t_{b, 2} /(1+z)$. Together with Eq. (10) and standard error propagation, one can roughly estimate $\tau_{\mathrm{c}, \mathrm{em}}>135 /(1+z)$ s. On the other hand, by assuming the efficiency $\eta=0.1, \eta L_{\mathrm{em}, 0} \simeq 4 \pi D_{L}^{2} F_{0}\left(D_{L}\right.$ is the luminosity distance) at redshift $z=0.01,0.1$, and 0.5 , one can estimate the upper limit of $B_{\mathrm{p}}$ and $P_{0}$ with equation of state GM1 (Lasky et al. 2014; Ravi \& Lasky 2014; Lü et al. 2015). The results are presented in Table 2. The comparison of the magnetar parameters with other short GRBs is shown in

Figure 4. The derived magnetar parameters of other short GRBs are taken from (Lü et al. 2015), they invoked the observed X-ray internal plateau of short GRBs to constrain magnetar parameters by assuming the energy loss from dipole radiation.

\section{GW radiation of magnetar}

If the energy loss of magnetar is dominated by GW radiation, one potential question is that how strong is the GW signal of magnetar. Based on the Eq.(4), one can derive the $\Omega(t)$ evolution as function of time (Lü et al. 2018),

$$
\Omega(t)=\Omega_{0}\left(1+\frac{t}{\tau_{\mathrm{c}, \mathrm{gw}}}\right)^{-1 / 4}
$$

and hence the GW frequency

$$
f(t)=f_{0}\left(1+\frac{t}{\tau_{\mathrm{c}, \mathrm{gw}}}\right)^{-1 / 4}
$$

where $f_{0}$ is initial GW frequency. So that, the amplitude of the GW signal decreases with time as (Fan et al. 2013; Lasky \& Glampedakis 2016; Lü et al. 2017)

$$
h_{\mathrm{c}}=\frac{1}{D_{\mathrm{L}}} \sqrt{\frac{5 G I f_{0}}{2 c^{3}}}\left(1+\frac{t}{\tau_{\mathrm{c}, \mathrm{gw}}}\right)^{-1 / 4}
$$

Here, the $h_{\mathrm{c}}$ is characteristic gravitational wave amplitude. The GW signal of new born magnetar at $t \sim 0$ is the strongest, so that the Eq.(13) can be approximate to following,

$$
h_{\mathrm{c}} \approx 8.22 \times 10^{-24}\left(\frac{I}{10^{45} \mathrm{~g} \mathrm{~cm}^{2}} \frac{f_{0}}{1 \mathrm{kHz}}\right)^{1 / 2}\left(\frac{D_{\mathrm{L}}}{100 \mathrm{Mpc}}\right)^{-1} \text {. }
$$


In Figure 5, we plot the GW strain sensitivity for advanced-LIGO (aLIGO, Aasi et al. 2015) and Einstein Telescope (ET, Punturo et al. 2010). It is clear that the GW strain of GRB $200219 \mathrm{~A}$ is below the aLIGO noise curve at $z=0.5$ and $z=0.1$, but it can be detected by current aLIGO at $z=0.01$. Abbott et al. (2017b) presented a search for GW emission from the remnant of the binary NS merger GW170817 using data from LIGO and Virgo within a short- and intermediate-time, but no GW signal from the post-merger remnant is found. From the theoretical point of view, the GW signal can be detected by current LIGO and Virgo if GRB 200219A is indeed located at $z=0.01$. Inspired by this point, we want to know whether this possible GW signal can be found out using data from LIGO and Virgo if GRB 200219A is located at $z=0.01$. By searching archived data from the LIGO website, we find that there is not any GW signal detected by LIGO in the interval two hours since the GRB trigger. This is an independent argument to show that the GRB 200219A cannot be located at $z=0.01$. Moreover, the signal may be detected by more sensitivity instruments at $z=0.01$ and $z=0.1$ in the future, such as ET.

\section{Possible merger-nova emission}

Neutron-rich ejecta can be powered by the merger of an NS binary, and heavier radioactive elements could be synthesized via r-process (Metzger 2017). Li \& Paczyński (1998) first calculated a near-isotropic signal in the optical/IR band that is powered by radioactive decay (without energy injection from the central engine). Yu et al. (2013) proposed that an optical/infrared transient can be powered by merger ejecta for the central magnetar, and this transient is brighter than that of in Li \& Paczyński (1998) due to an additional source of sustained energy injection from the magnetar, they called it merger-nova (Yu et al. 2013; Gao et al. 2017). If this is the case, one interesting question is how bright this merger-nova is? In this section, following the method of $\mathrm{Yu}$ et al. (2013), we roughly calculate merger-nova emission at different distances. We adopt the following parameters which are from the first double NSs merger event (GW170817/GRB 170817A) by fitting the light curve of AT2017gfo, so that the parameters of merger-nova we used are from $\mathrm{Yu}$ et al. (2018) and Hajela et al. (2019), e.g., ejecta mass $M_{e j}=10^{-2} M_{\odot}$, velocity $\beta_{e j}=0.1 \mathrm{c}$, opacity $\kappa=0.97 \mathrm{~cm}^{2} \mathrm{~g}^{-1}$, medium density $n=0.01 \mathrm{~cm}^{-3}$, as well as the spin-down timescale roughly equal to $t_{b, 2}$.

Figure [ shows the possible merger-nova light curve of GRB 200219A by only considering the contribution of the magnetar-powered in $\mathrm{K}-, \mathrm{r}-$, and $\mathrm{U}$-band at $z=0.01,0.1$, and 0.5 . Moreover, we also overplot the upper limit detected of instruments in Figure 6, e.g., Large 
Synoptic Survey Telescope(LSST), normal-LSST7, PTF, and Pan-STARRS (Jedicke et al. 2007; Law et al. 2009; Jones et al. 2009; Metzger \& Berger 2012). The numerical calculation shows that the merger-nova is bright enough to be detected by all instruments above at $z=0.01$. However, it is a little bit $\operatorname{dim}$ at $z=0.1$ and 0.5 . More details of systematically searching and calculations with measured redshift short GRBs are presented in Yuan et al. (2020).

\section{Discussion}

In the previous works, the observed steep decay (decay slope typically $3 \sim 5$ ) after prompt emission can be interpreted as the curvature effect, which is the delay of propagation of photons from high latitudes with respect to the line of sight (Fenimore et al. 1996; Kumar \& Panaitescu 2000; Liang et al. 2006; Zhang et al. 2007). If this is the case, the predicted temporal decay index and spectral index of the emission satisfy with a relation,

$$
\alpha=2+\beta
$$

In order to test this possibility, we extract the time-average spectrum of X-ray during the abrupt drop phase with the power-law model. One has $\beta=\Gamma-1=0.87 \pm 0.22$, where $\Gamma=1.87 \pm 0.22$ is the power-law index of spectral fitting. It is easy to check that it is not consistent with above predicted correlation. Moreover, the plateau emission followed by a $\sim t^{-1}$ decay phase are also inconsistent with the curvature effect.

Alternatively, the sharp decay of X-ray light curve in the Swift era was usually interpreted as jet break, which is geometric effect when the fireball decelerate with the beaming angle eventually exceeding the physical collimation angle (Liang et al. 2008; Racusin et al. 2009). If this sudden decrease in the flux at $t=200$ seconds after trigger is caused by jet break, the break time is much shorter than that of Swift GRBs observed jet break which are as long as $10^{4} \sim 10^{5} \mathrm{~s}$ (Liang et al. 2008; Racusin et al. 2009). On the other hand, a normal decay phase with temporal index $\alpha \sim 1.04$ is observed again after the sharp decay, this segment should not appear if the sharp decay is interpreted with jet break.

By systematically searching all short GRBs observed with Swift, we find that another two short GRBs with redshift measured are also consistent with the magnetar central engine, but the physical process is different from GRB 200219A. (1) One is GRB 050724 with EE

\footnotetext{
${ }^{7}$ The expected maximum depth of LSST and normal-LSST are about 26.5 and 24.7 magnitude, respectively. It means that the LSST should be better to observe more dim image within a longer exposure time (Metzger \& Berger 2012).
} 
at redshift $z=0.258$ (Barthelmy et al. 2005), its early X-ray light curve presents a plateau emission followed by a $\sim t^{-2}$ decay phase, then continue to an abrupt drop segment $\left(\sim t^{-8}\right)$. It is natural to explain by invoking supramassive magnetar from the merger of an NS binary. The magnetar spins-down losing its rotation energy mostly via the EM channel and then collapse into a black hole after surviving hundreds of seconds. (2) Another one is nearby GRB 160821B with $z=0.16$ (Levan et al. 2016); its early X-ray light curve shows a plateau emission followed by an abrupt drop decay $\left(\sim t^{-4.5}\right)$. However, there is no signature revealing that whether the collapse is caused by EM or GW dominated radiation, more details also see Lü et al. (2017). A comparison of the X-ray light curves of those three short GRBs are shown in Figure 7. These results suggest that at least a supramassive NS/magnetar can survive in the central engine of some short GRBs, and it spins down via losing its rotation energy due to either GW-dominated radiation or EM-dominated radiation. Moreover, Sarin et al. (2019) found that the millisecond magnetar model is favoured over the fireball model for two short GRBs by analyzing its X-ray data, but it is dependent on the unknown equation of state and non-rotating neutron star mass. The more robust evidence of this hypothesis is to catch such weak GW signal from supramassive magnetar by a-LIGO and Virgo in the future.

\section{Conclusions}

GRB 200219A is a short GRB with duration less than 1 s, observed by both Swift and Fermi. The extended emission component lasting $\sim 90 \mathrm{~s}$ after the initial hard spike is identified by the Swift/BAT, but it is not significant in the Fermi/GBM temporal analysis. We presented a broadband analysis of its prompt and afterglow emission and found that the peak energy of its spectrum is as high as $1387_{-134}^{+232} \mathrm{keV}$, which is harder than most short GRBs observed by Fermi/GBM. More interestingly, together with the EE component and early X-ray data, a plateau emission was followed by a $\sim t^{-1}$ segment, then with an extremely steep decay. This early temporal feature is very difficult to explain with the standard internal/external shock model of a black hole central engine, but could be consistent with the prediction of a magnetar central engine from the merger of an NS binary. We explain the plateau emission followed by a $\sim t^{-1}$ decay phase from spinning down of millisecond magnetar, which loses its rotation energy via GW quadrupole radiation. Then, the magnetar collapsing into the black hole before switching to the EM-dominated is corresponding to abrupt drop decay.

However, a fly in the ointment is no redshift measured of this case, so we have to attempt the pseudo redshift to reveal its physical properties. Several numerical calculations 
are summarized as following:

- By assuming that the pseudo redshift $z=0.01$ which is the approximate luminosity distance as GW170817/GRB 170817A event. The requirements of physical parameters of magnetar are not reasonable, especially, $B_{p}$ is as high as $10^{17} \mathrm{G}$. The signal of GW radiation of central magnetar at this distance is high enough and can be detected by current a-LIGO and ET in the future. Moreover, at a later time, the peak luminosity of possible merger-nova is also above the upper limit detected of several instruments.

- If the pseudo redshift $z=0.1$, that is close to the upper limit of GW signal detected by current LIGO/Virgo. The physical parameters of magnetar seem to be in a reasonable range. The signal of GW at this distance is below the noise curve of current a-LIGO, but is expected to detect by ET in the future. The merger-nova signal is also potential to be detected by survey telescopes in the future.

- If we adopt the pseudo redshift $z=0.5$, which is the central value of the redshift distribution for all short GRBs with $z$ measurements, this requires a rapidly rotating magnetar with a spin period $\sim 1 \mathrm{~ms}$, and a surface magnetic field in a reasonable range. The GW signal cannot be detected by a-LIGO or ET. The merger-nova signal at this distance can be comparable with the limits from some optical survey telescopes.

We acknowledge the use of public data from the Swift and Fermi data archive, and the UK Swift Science Data Center. This work is supported by the National Natural Science Foundation of China (grant Nos.11922301, 11851304, 11533003, and 11833003), the Guangxi Science Foundation (Grant Nos. 2017GXNSFFA198008, 2018GXNSFGA281007, and AD17129006). The One-Hundred-Talents Program of Guangxi colleges, Bagui Young Scholars Program (LHJ), and special funding for Guangxi distinguished professors (Bagui Yingcai \& Bagui Xuezhe). BBZ acknowledges support from a national program for young scholars in China, Program for Innovative Talents and Entrepreneur in Jiangsu, and a National Key Research and Development Programs of of China (2018YFA0404204).

\section{REFERENCES}

Aasi, J., Abadie, J., Abbott, B. P., et al. 2015, Classical and Quantum Gravity, 32, 115012 Abbott, B. P., Abbott, R., Abbott, T. D., et al. 2017a, ApJ, 848, L13

Abbott, B. P., Abbott, R., Abbott, T. D., et al. 2017b, ApJ, 851, L16 
Amati, L., Frontera, F., Tavani, M., et al. 2002, A\&A, 390, 81

Barthelmy, S. D., Chincarini, G., Burrows, D. N., et al. 2005, Nature, 438, 994

Berger, E. 2014, ARA\&A, 52, 43

Bissaldi, E., \& Meegan, C. 2020, GRB Coordinates Network 27137, 1

Bucciantini, N., Metzger, B. D., Thompson, T. A., et al. 2012, MNRAS, 419, 1537

Chen, W., Xie, W., Lei, W.-H., et al. 2017, ApJ, 849, 119

Corsi, A., \& Mészáros, P. 2009, ApJ, 702, 1171

Dai, Z. G., \& Lu, T. 1998a, A\&A, 333, L87

Dai, Z. G., \& Lu, T. 1998b, Phys. Rev. Lett., 81, 4301

Dai, Z. G., Wang, X. Y., Wu, X. F., et al. 2006, Science, 311, 1127

Eichler, D., Livio, M., Piran, T., et al. 1989, Nature, 340, 126

Evans, P. A., Beardmore, A. P., Page, K. L., et al. 2009, MNRAS, 397, 1177

Fan, Y.-Z., Yu, Y.-W., Xu, D., et al. 2013, ApJ, 779, L25

Fenimore, E. E., Madras, C. D., \& Nayakshin, S. 1996, ApJ, 473, 998

Gao, H., Zhang, B., \& Lü, H.-J. 2016, Phys. Rev. D, 93, 044065

Gao, H., Zhang, B., Lü, H.-J., et al. 2017, ApJ, 837, 50

Gao, W.-H., \& Fan, Y.-Z. 2006, Chinese Journal of Astronomy and Astrophysics, 6, 513

Giacomazzo, B., \& Perna, R. 2013, ApJ, 771, L26

Goldstein, A., Veres, P., Burns, E., et al. 2017, ApJ, 848, L14

Hajela, A., Margutti, R., Alexander, K. D., et al. 2019, ApJ, 886, L17

Jedicke, R., Magnier, E. A., Kaiser, N., et al. 2007, Near Earth Objects, Our Celestial Neighbors: Opportunity and Risk, 341

Jones, R. L., Chesley, S. R., Connolly, A. J., et al. 2009, Earth Moon and Planets, 105, 101

Kumar, P., \& Panaitescu, A. 2000, ApJ, 541, L51 
Kumar, P., \& Zhang, B. 2015, Phys. Rep., 561, 1

Laha, S., Barthelmy, S. D., Cummings, J. R., et al. 2020, GRB Coordinates Network 27148, 1

Lasky, P. D., Haskell, B., Ravi, V., et al. 2014, Phys. Rev. D, 89, 047302

Lasky, P. D., \& Glampedakis, K. 2016, MNRAS, 458, 1660

Law, N. M., Kulkarni, S. R., Dekany, R. G., et al. 2009, PASP, 121, 1395

Lei, W. H., Wang, D. X., Zhang, L., et al. 2009, ApJ, 700, 1970

Levan, A. J., Wiersema, K., Tanvir, N. R., et al. 2016, GRB Coordinates Network 19846, 1

Li, A., Zhang, B., Zhang, N.-B., et al. 2016, Phys. Rev. D, 94, 083010

Li, L.-X., \& Paczyński, B. 1998, ApJ, 507, L59

Li, L., Liang, E.-W., Tang, Q.-W., et al. 2012, ApJ, 758, 27

Liang, E. W., Zhang, B., O’Brien, P. T., et al. 2006, ApJ, 646, 351

Liang, E.-W., Racusin, J. L., Zhang, B., et al. 2008, ApJ, 675, 528

Lien, A. Y., Ambrosi, E., D’Ai, A., et al. 2020, GRB Coordinates Network 27125, 1

Liu, T., Gu, W.-M., \& Zhang, B. 2017, NewAR, 79, 1

Lu, R.-J., Du, S.-S., Cheng, J.-G., et al. 2017, arXiv e-prints, arXiv:1710.06979

Lyons, N., O'Brien, P. T., Zhang, B., et al. 2010, MNRAS, 402, 705

Lü, H.-J., \& Zhang, B. 2014, ApJ, 785, 74

Lü, H.-J., Zhang, B., Lei, W.-H., et al. 2015, ApJ, 805, 89

Lü, H.-J., Zhang, H.-M., Zhong, S.-Q., et al. 2017, ApJ, 835, 181

Lü, H.-J., Zou, L., Lan, L., et al. 2018, MNRAS, 480, 4402

Meegan, C., Lichti, G., Bhat, P. N., et al. 2009, ApJ, 702, 791

Metzger, B. D., Giannios, D., Thompson, T. A., et al. 2011, MNRAS, 413, 2031

Metzger, B. D., \& Berger, E. 2012, ApJ, 746, 48 
Metzger, B. D., \& Piro, A. L. 2014, MNRAS, 439, 3916

Metzger, B. D. 2017, Living Reviews in Relativity, 20, 3

O’Brien, P. T., Willingale, R., Osborne, J., et al. 2006, ApJ, 647, 1213

Paczynski, B. 1986, ApJ, 308, L43

Paczynski B., 1991, AcA, 41, 257

Popham, R., Woosley, S. E., \& Fryer, C. 1999, ApJ, 518, 356

Punturo, M., Abernathy, M., Acernese, F., et al. 2010, Classical and Quantum Gravity, 27, 194002

Racusin, J. L., Liang, E. W., Burrows, D. N., et al. 2009, ApJ, 698, 43

Ravi, V., \& Lasky, P. D. 2014, MNRAS, 441, 2433

Roming, P. W. A., Kennedy, T. E., Mason, K. O., et al. 2005, Space Sci. Rev., 120, 95

Rosswog, S., Ramirez-Ruiz, E., \& Davies, M. B. 2003, MNRAS, 345, 1077

Rowlinson, A., O’Brien, P. T., Tanvir, N. R., et al. 2010, MNRAS, 409, 531

Rowlinson, A., O’Brien, P. T., Metzger, B. D., et al. 2013, MNRAS, 430, 1061

Sakamoto, T., Barthelmy, S. D., Barbier, L., et al. 2008, ApJS, 175, 179

Sarin, N., Lasky, P. D., \& Ashton, G. 2019, ApJ, 872, 114

Savchenko, V., Ferrigno, C., Kuulkers, E., et al. 2017, ApJ, 848, L15

Shapiro, S. L., \& Teukolsky, S. A. 1983, A Wiley-Interscience Publication

Siegel, M. H., Lien, A. Y., \& Swift/UVOT Team 2020, GRB Coordinates Network 27165, 1

Sowell, E., Corsi, A., \& Coyne, R. 2019, Phys. Rev. D, 100, 124041

Thompson, C. 1994, MNRAS, 270, 480

Troja, E., Cusumano, G., O’Brien, P. T., et al. 2007, ApJ, 665, 599

Usov, V. V. 1992, Nature, 357, 472

Wheeler, J. C., Yi, I., Höflich, P., et al. 2000, ApJ, 537, 810 
Yu, Y.-W., Zhang, B., \& Gao, H. 2013, ApJ, 776, L40

Yu, Y.-W., Liu, L.-D., \& Dai, Z.-G. 2018, ApJ, 861, 114

Zhang, B.-B., Zhang, B., Sun, H., et al. 2018a, Nature Communications, 9, 447

Zhang, B.-B., Zhang, B., Castro-Tirado, A. J., et al. 2018b, Nature Astronomy, 2, 69

Zhang, B.-B., Zhang, B., Liang, E.-W., et al. 2011, ApJ, 730, 141

Zhang, B.-B., Uhm, Z. L., Connaughton, V., et al. 2016, ApJ, 816, 72

Zhang, B.-B., Liang, E.-W., \& Zhang, B. 2007, ApJ, 666, 1002

Zhang, B., \& Mészáros, P. 2001, ApJ, 552, L35

Zhang, B., Zhang, B.-B., Virgili, F. J., et al. 2009, ApJ, 703, 1696

Zhang, B. 2013, ApJ, 763, L22

This preprint was prepared with the AAS IATEX macros v5.2. 
Table 1. Curved Power-law spectral fit parameters for the Fermi/GBM $T_{90}$ data of GRB $200219 \mathrm{~A}$

\begin{tabular}{cccccc}
\hline \hline Time Interval & \multicolumn{5}{c}{ CPL } \\
\hline$t_{s}$ & $t_{e}$ & $\Gamma_{\mathrm{ph}}$ & $E_{\mathrm{p}}$ & $\log$ Norm & PGSTAT/dof \\
\hline-0.02 & 0.5 & $-0.65 \pm 0.07$ & $1387_{-134}^{+232}$ & $-0.19_{-0.15}^{+0.13}$ & $278 / 351$ \\
\hline
\end{tabular}

Table 2. The derived parameters of magnetar for different redshift

\begin{tabular}{ccccc}
\hline \hline $\begin{array}{c}\text { Redshift } \\
(z)\end{array}$ & $\begin{array}{c}L_{\text {em }, 0} \\
\left(\mathrm{erg} \mathrm{s}^{-1}\right)\end{array}$ & $\begin{array}{c}B_{p} \\
(\mathrm{G})\end{array}$ & $\begin{array}{c}P_{0} \\
\left(10^{-3} \mathrm{~s}\right)\end{array}$ & $h_{c}{ }^{\mathrm{a}}$ \\
\hline 0.01 & $(1.09 \pm 0.24) \times 10^{46}$ & $<4.6 \times 10^{17}$ & $<118$ & $1.96 \times 10^{-23}$ \\
0.1 & $(1.30 \pm 0.31) \times 10^{48}$ & $<4.3 \times 10^{16}$ & $<11.2$ & $1.79 \times 10^{-24}$ \\
0.5 & $(4.92 \pm 1.13) \times 10^{49}$ & $<9.4 \times 10^{15}$ & $<2.1$ & $2.91 \times 10^{-25}$ \\
\hline
\end{tabular}

aThe GW strain of magnetar for initial $f_{0}=1000 \mathrm{~Hz}$.
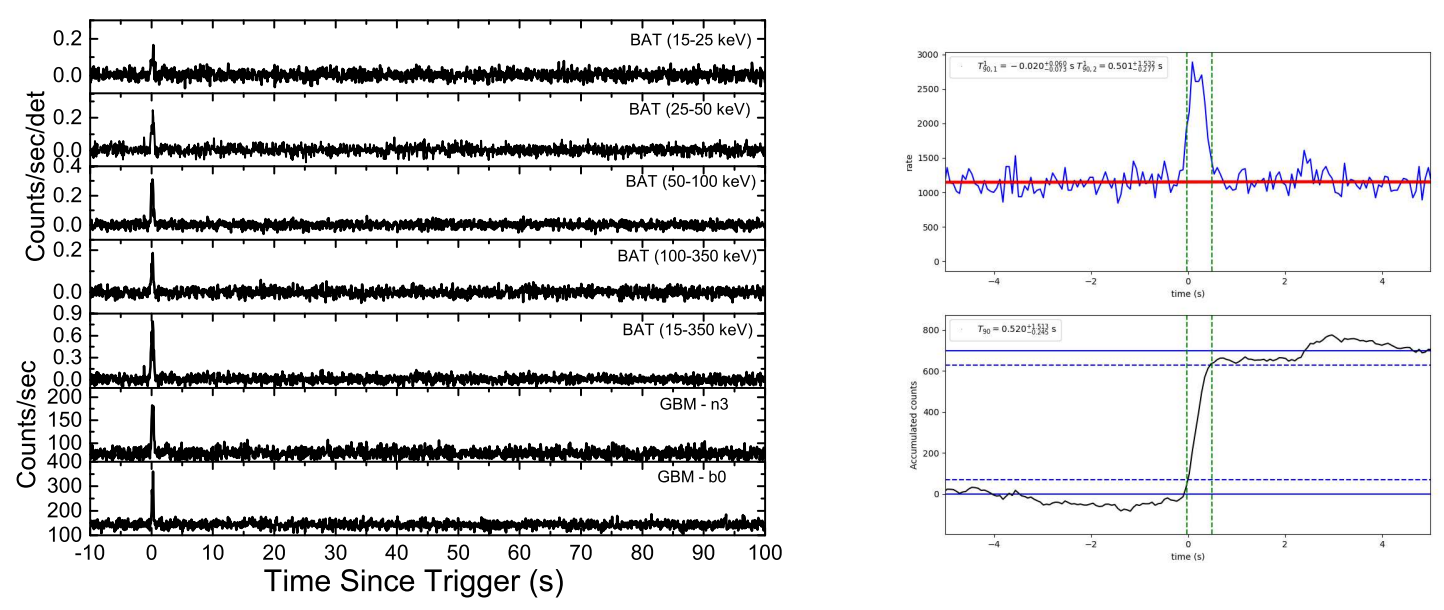

Fig. 1.- Left:Swift/BAT and Fermi/GBM light curves of GRB 200219A in different energy bands with a $128 \mathrm{~ms}$ time bin. Right: The determination of its $T_{90}$ for Fermi/GBM. Green horizontal dashed lines are shown $5 \%$ and $95 \%$ of accumulated counts. Vertical dotted lines are drawn at the times corresponding to accumulated counts, which are used to define the $T_{90}$ intervals. 

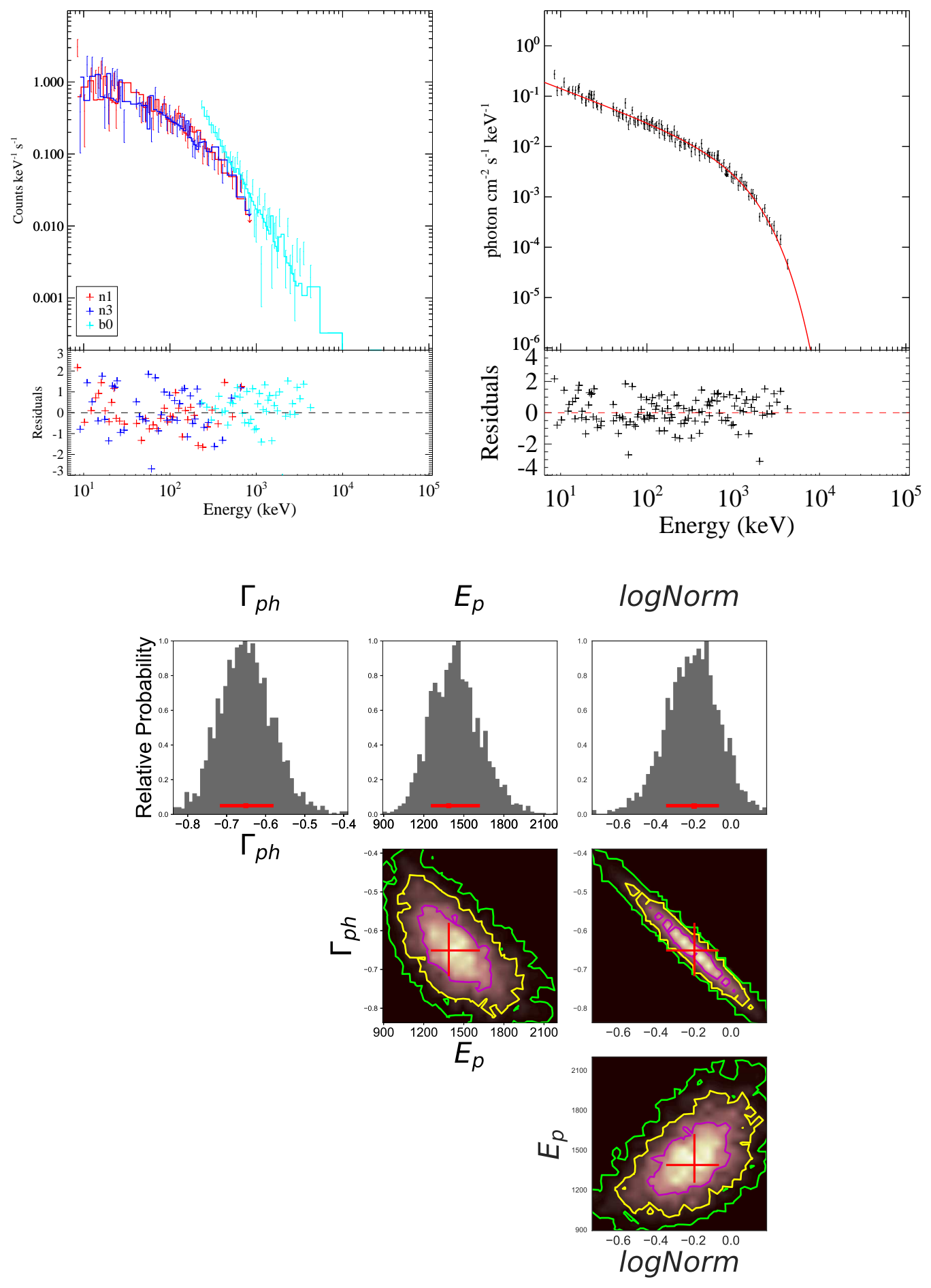

Fig. 2. - The spectral fits of GRB 200219A with cutoff power-law model for Fermi/GBM $T_{90}$. The count spectrum (Top left), photon spectrum (Top right), as well as parameter constraints of the CPL fit. Histograms and contours in the corner plots show the likelihood map of constrained parameters by using our McSpecFit package. Red crosses are the bestfitting values, and pink, yellow, and green circles are the $1 \sigma, 2 \sigma$, and $3 \sigma$ uncertainties, respectively. 

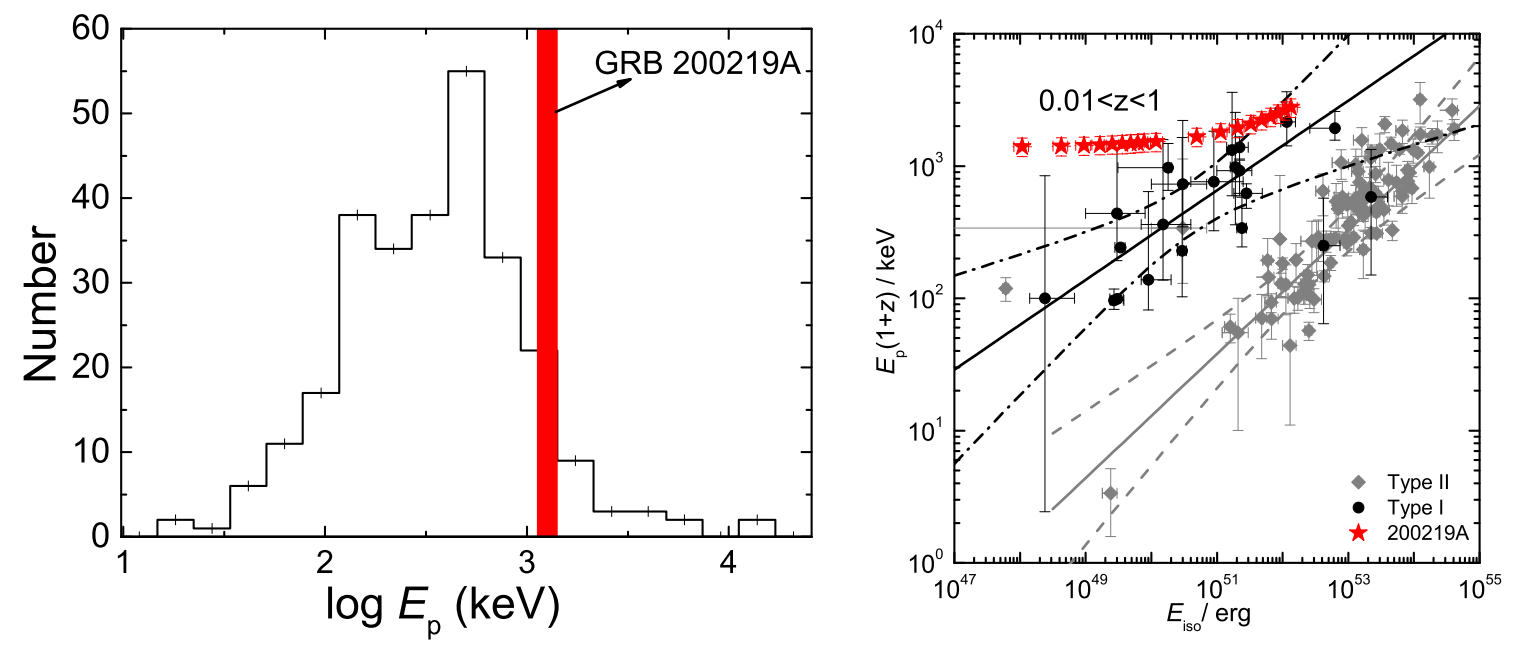

Fig. 3.- Left: $E_{\mathrm{p}}$ distribution for GRB 200219A and other short GRBs observed by Fermi/GBM. The $E_{\mathrm{p}}$ values of other short GRBs are taken from Lu et al. (2017). Right: $E_{\mathrm{p}}$ and $E_{\text {iso }}$ correlation diagram. Black points and grey diamonds are corresponding to Type I and Type II GRBs, which are taken from Zhang et al. (2009). The red stars are the GRB 200219A with pseudo redshift from 0.01 to 1 . The redshift step is 0.01 from $z=0.01$ to 0.1 , and with step 0.1 from $z=0.1$ to 1.0. The best-fit $E_{\mathrm{p}}-E_{\text {iso }}$ correlations for both Type II (grey diamonds) and Type I (black points) GRBs are plotted (solid lines) with the $3 \sigma$ boundary (dashed line) marked.
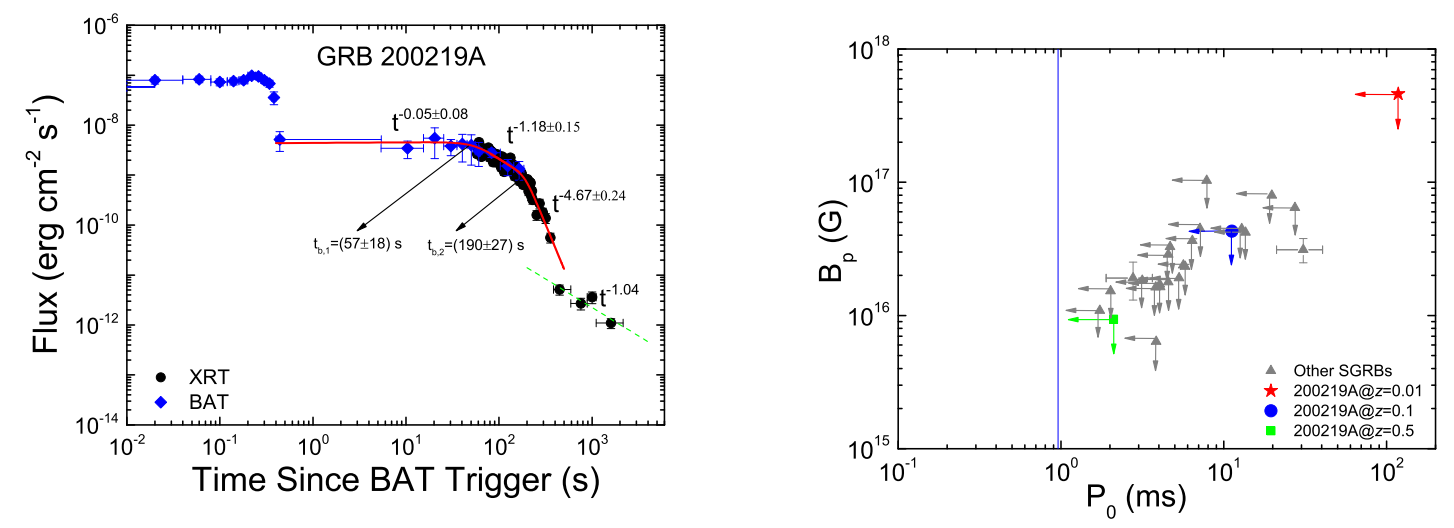

Fig. 4. - Left: X-ray light curve in (0.3-10) keV and empirical fit with a smoothed triple power law model. right: Inferred magnetar parameters $\left(P_{0}\right.$ vs. $\left.B_{p}\right)$ of GRB 200219A with $z=0.01$ (red star), 0.1 (blue dot), and 0.5 (green square) compared with other short GRBs (gray triangle). The derived magnetar parameters of other short GRBs are taken from Lü et al. (2015). Vertical solid line is the break-up spin period limit of neutron star. 


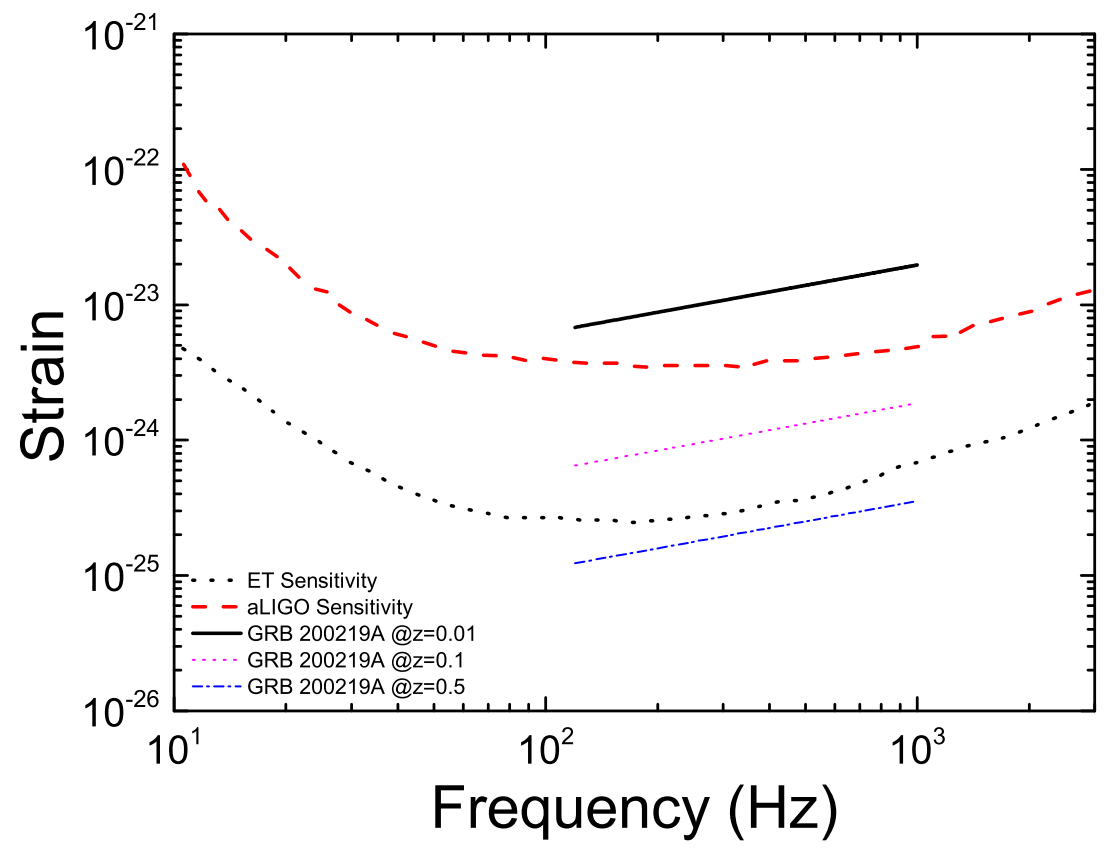

Fig. 5.- GW strain evolution with frequency for GRB 200219A at pseudo redshift $z=0.01$ (black solid line), $z=0.1$ (pink dotted line), and $z=0.5$ (blue dashCdotted line). The black dotted line and red dashed line are the sensitivity limits for aLIGO and ET, respectively.
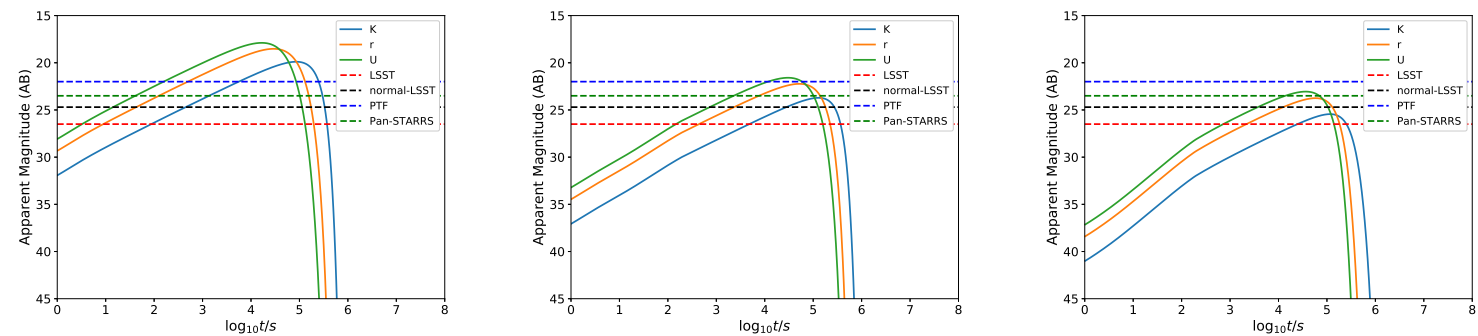

Fig. 6. - Merger-nova light curve of GRB 200219A by only considering the contribution of the magnetar-powered in $\mathrm{K}-, \mathrm{r}-$, and U-band at $z=0.01$ (left), 0.1 (middle), and 0.5 (right). The horizontal dotted lines are corresponding to upper limit detected of normalLSST (black), PTF (blue), Pan-STARRS (green), and LSST (red) surveys, respectively. 


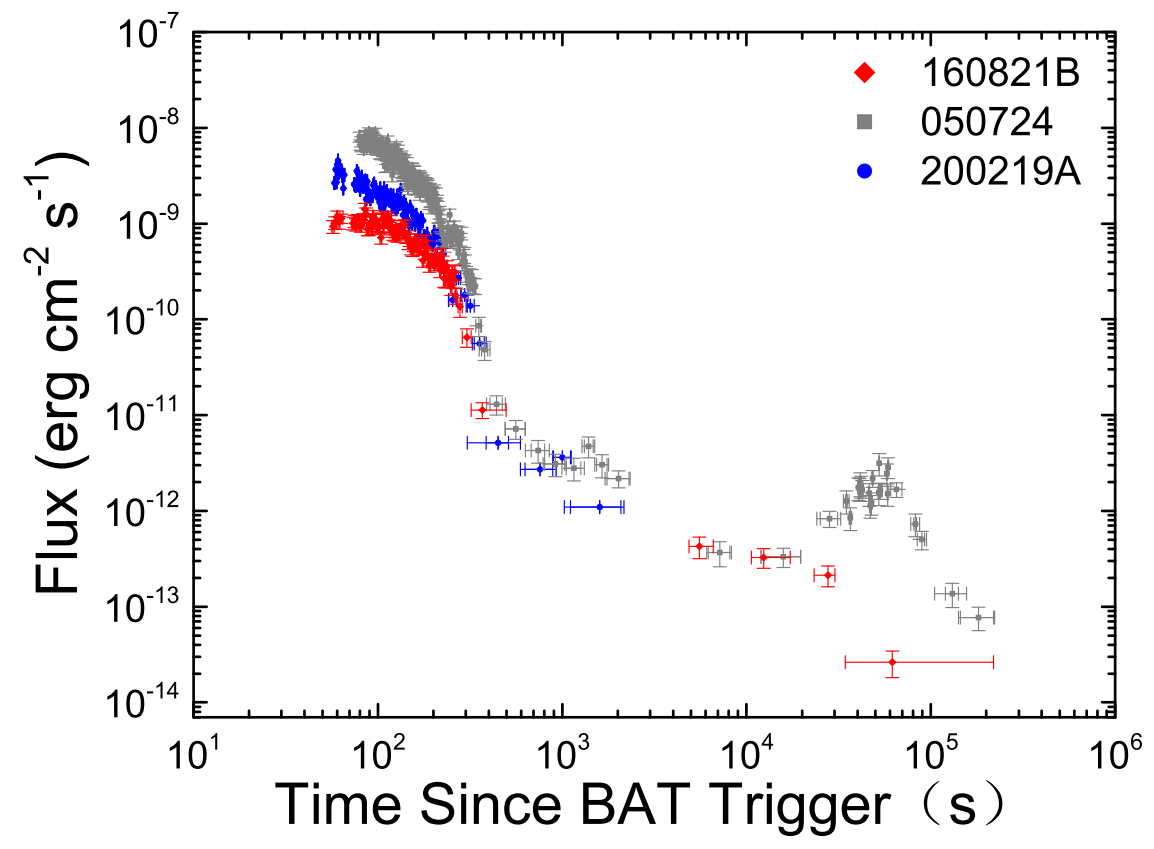

Fig. 7.- Comparison X-ray light curves of GRB 200219A with GRBs 050724 and 160821B in (0.3-10) keV. The X-ray data of GRBs 050724 and 160821B are taken from Swift/XRT webset: https : //www.swift.ac.uk/xrt_curves/allcurves.php. 